\title{
The package as a weapon of influence: Changes to cigarette packaging design as a function of regulatory changes in Canada
}

\author{
Wade S. Wade ${ }^{1}$ Katherine White ${ }^{1}$
}

\begin{abstract}
INTRODUCTION Given existing regulations that ban the tobacco industry from engaging in traditional forms of advertising and require warning labels on cigarette packaging, we suggest that one response on the part of tobacco manufacturers has been to make alterations to design elements of cigarette packages themselves. The current research seeks to examine how cigarette manufacturers have altered elements of cigarette packaging in response to regulatory changes by the Government of Canada in 2011, which increased health warning sizes on cigarette packages from $50 \%$ of the principal display surface to $75 \%$.

METHODS Cigarette packages $(n=1689)$ that had been on the market in Canada in the period 2001-2017 were examined and coded for package design elements including package innovation (size and package style), color (hue and saturation), and branding elements (use of iconography and variant names). Characteristics of pre-regulation packaging were then systematically compared to characteristics of post-regulation packaging.

RESULTS Many of these packaging design elements, including package size and package style, primary and secondary hue, color saturation, use of variant label names, and use of iconography have systematically varied in response to regulatory changes in Canada. For example, we observed increases in the use of flip-top (vs slide and shell) packaging, the use of yellow, black and white as the focal color, incidence of color-themed variant names, and the use of female and crest-related logos.

CONCLUSIONS The evidence suggests that many packaging design elements have varied systematically along with regulatory changes in Canada.
\end{abstract}

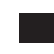

\section{AFFILIATION \\ 1 Marketing and Behavioral Sciences Department, Sauder School of Business, University of British Columbia, Vancouver, Canada}

\section{CORRESPONDENCE TO}

Katherine White. Marketing and Behavioral Sciences Department, Sauder School of Business, University of British Columbia, 2329 West Mall, Vancouver V6T 1Z4, Canada. E-mail:

katherine.white@sauder.ubc.ca

\section{KEYWORDS}

tobacco, packaging, policy, tobacco control, cigarettes

Received: 1 November 2019 Revised: 9 January 2020

Accepted: 20 January 2020

\section{INTRODUCTION}

Governments worldwide have gone to great lengths to discourage the consumption of tobacco products ${ }^{1}$. Despite these efforts, in most countries tobacco companies are still able to use their packages as a way to differentiate their brand and appeal to consumers ${ }^{2}$. Recognizing the importance of cigarette packaging, many governments have established regulations that limit the packaging elements that can be used by tobacco companies $^{3}$, and mandated the display of graphic health warnings on packages ${ }^{4,5}$. In this study, we review the literature on tobacco packaging as a promotional tool and present data from 1689 cigarette packages that were on the market in Canada between 2001-2017. Our objective is to understand how regulatory changes by the Government of Canada relate to variations in packaging design elements over time.

Canada has implemented several tobacco regulations. As a result, the prevalence of cigarette smoking in Canada fell from $25 \%$ to $13 \%$ in the 
period $1999-2015^{6}$. However, smoking remains the number one cause of preventable death in Canada and is a relevant health concern for the nation's 4.6 million smokers ${ }^{7}$. In 2011, the Canadian government passed an Act called the Tobacco Products Labeling Regulations-Cigarettes and Little Cigars (TPLRCLC). The TPLR-CLC requires that all tobacco packages display a graphic health warning covering $75 \%$ of the surface of the principal display area of all tobacco packages, up from $50 \%$ in 2018 . In addition, this regulation mandated the display of a tollfree quitline and a web address to access smoking cessation services. We suggest that, because cigarette manufacturers now have increasingly limited means of promoting their products, one response to such regulations has been to change package design elements in ways that make the product more appealing to consumers.

The tobacco industry has been known to innovate several packaging elements to attract consumers ${ }^{9}$. These include, but are not limited to, package style, number of cigarettes per package, variant names, iconography, color, and color saturation. Tobacco companies manipulate these packaging elements in an effort to segment markets, create positive associations with their products, and influence consumer perceptions ${ }^{2,10}$. The extant research suggests that tobacco companies might use package design elements to promote their products to consumers ${ }^{2}$. Additionally, research shows that government regulations have severely limited the promotional activities that tobacco companies can engage in ${ }^{11}$. We build on this research to examine whether tobacco manufacturers have made changes to tobacco packaging on a timeline that corresponds with the TPLR-CLC. Given that tobacco companies' promotional outlets have been restricted, analyzing tobacco packaging before and after the TPLR-CLC was passed should provide insights into how tobacco companies have altered their packaging over time. We note that other regulations might have impacted changes in cigarette packaging over time. We focused on the TPLR-CLC because Health Canada viewed this as being a major and influential regulation.

\section{METHODS}

In this research, we assessed a sample of 1689 tobacco packages from 92 brands that were curated by Health
Canada. We assessed the changes that have occurred to tobacco packages before (T1) and after (T2) the TPLR-CLC was passed. There were 1202 packages from $\mathrm{T} 1$ and 487 from $\mathrm{T} 2$. The assessment was carried out by two independent coders, with disagreements resolved via discussion. The coders analyzed the packages based on package style $(\mathrm{Kappa}=0.897)$ and package size $(\mathrm{Kappa}=0.902)$. The coders also analyzed the focal hue or the color that took up most of the available surface area $(\mathrm{Kappa}=0.893)$, the secondary hue or the color that took up the secondmost surface area (Kappa=0.911), the degree of saturation of the focal hue, defined as the intensity or purity of the hue $(\mathrm{r}=0.878)$, and the degree of saturation of the secondary hue $(r=0.913)$, variant names (Kappa=0.972), and package iconography, including logos and images (Kappa=0.931). A table displaying the coding protocol and detailed statistical analyses for all of our measured variables can be found in the Supplementary file.

\section{RESULTS}

A chi-squared analysis shows significant differences in package style between $\mathrm{T} 1$ and $\mathrm{T} 2\left(\chi^{2}(3)=101.06\right.$; $\mathrm{p}<0.0001)$. During $\mathrm{T} 1$, the majority of packages (62.2\%) were slide and shell packages, followed by flip-top (36.4\%), soft packaging (1.4\%), and slim design $(<1 \%)$. During T2, most packages were flip-top style $(57.1 \%)$, followed by slide and shell $(40.5 \%)$, slim design $(2.5 \%)$ and soft packaging $(<1 \%)$. Across T1 and T2, the incidence of flip-top increased significantly, $\left(X^{2}(1)=61.00 ; p<0.0001\right)$ while the incidence of the slide and shell decreased significantly $\left(X^{2}(1)=66.69 ; p<0.0001\right)$. The average number of cigarettes per package decreased from 25.16 in $\mathrm{T} 1$ to 22.21 in $\mathrm{T} 2(\mathrm{t}(18)=3.03 ; \mathrm{p}=0.002)$. Significant changes in the focal hue were observed across T1 and T2 $\left(\chi^{2}(10)=672 ; \mathrm{p}<0.0001\right)$, as shown in Figure 1. According to pairwise chi-squared tests, the use of blue, red and grey/silver decreased from T1 to T2 $\left(X^{2} \mathrm{~s}>35 ; \mathrm{ps}<0.001\right)$, while the use of white, black and yellow increased $(x 2 s>13 ; p s<0.001)$. The use of secondary colors also changed pre- and postregulation $\left(X^{2}(11)=569.63 ; p<0.0001\right)$. Pairwise chisquare tests indicate that the use of blue, red and yellow as secondary hues increased $\left(\chi^{2} \mathrm{~s}>36\right.$; $\left.\mathrm{ps}<0.001\right)$ while the use of white and black as secondary hues decreased $\left(\chi^{2} \mathrm{~s}>104 ; \mathrm{ps}<0.001\right)$ from $\mathrm{T} 1$ to $\mathrm{T} 2$. 
Figure 1. Proportions of Focal Package Hues Pre- and Post-Regulation

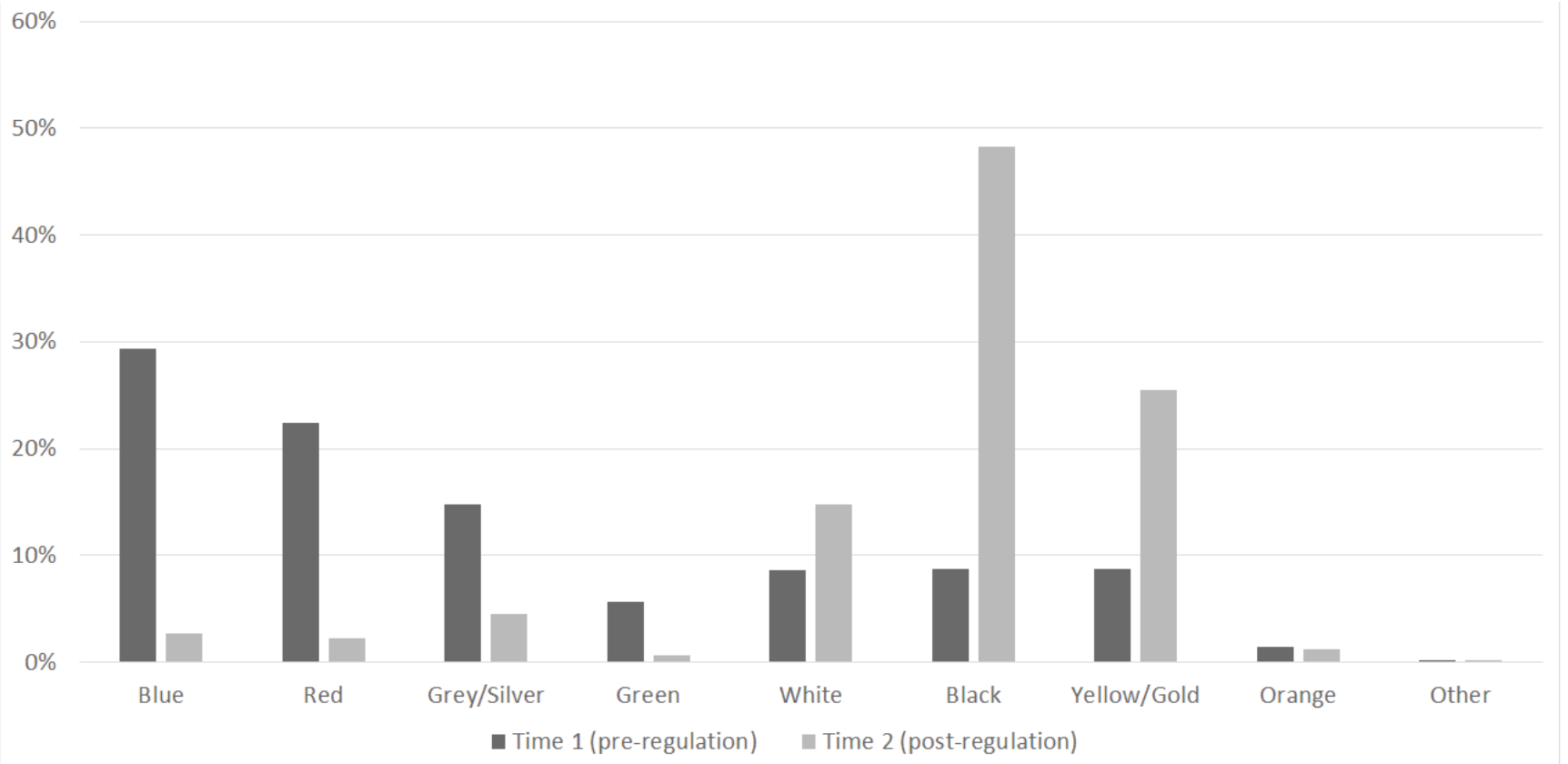

We also observed a substantial increase in the use of high focal hue saturation during T2 $\left(\chi^{2}(2)=429.17 ; p<0.0001\right)$. Pre-regulation, high color saturation was only found in $35.8 \%$ of packaging. Post-regulation, $91.4 \%$ of packaging contained high color saturation. For secondary color saturation, there was a significant increase in the use of high, rather than medium, color saturation after the packaging regulations were implemented $\left(X^{2}(1)=428.49 ; \mathrm{p}<0.0001\right)$. Pre-regulation, $16.5 \%$ of the background colors contained low color saturation, $47.8 \%$ contained medium color saturation, and $35.8 \%$ contained high color saturation. In contrast, post-regulation background colors contained on average $2.1 \%$ low saturation, $6.6 \%$ medium saturation, and $91.4 \%$ high saturation.

The use of variants changed from T1 to T2 $\left(\chi^{2}(6)=126.68 ; p<0.0001\right)$. There was a decrease in flavor variant labels from $43.1 \%$ to $30.3 \%\left(\chi^{2}(1)=23.53\right.$; $\mathrm{p}<0.0001)$, as well as the disappearance of light or mild labels, from $9 \%$ to $0 \%\left(X^{2}(1)=46.07\right.$; $\left.p<0.0001\right)$. Additionally, the use of color variants increased substantially from $12.8 \%$ to $29.4 \%\left(X^{2}(1)=65.16\right.$; $\mathrm{p}<0.0001)$. The use of no variant also increased over time from $13 \%$ to $18.8 \%\left(\chi^{2}(1)=9.18 ; p=0.0024\right)$.

The use of iconography changed significantly between T1 and T2 $\left(\chi^{2}(1)=218.3 ; \mathrm{p}<0.0001\right)$. Male figures in brand logos almost disappeared, from $35.1 \%$ pre-regulation to $1.4 \%$ post-regulation $\left(X^{2}(1)=205.69 ; p<0.0001\right)$, while the use of women in brand logos increased from $7.8 \%$ to $11.2 \%$ $\left(X^{2}(1)=4.85 ; p=0.0276\right)$. The use of crests increased substantially, from $29 \%$ to $44.1 \%\left(\chi^{2}(1)=35.18\right.$; $\mathrm{p}<0.0001)$. In addition, the number of brands with no logo or iconography increased from $18.6 \%$ to $32.5 \%\left(\chi^{2}(1)=37.88 ; p<0.0001\right)$.

\section{DISCUSSION}

The results of this research provide evidence of systematic changes to tobacco packaging corresponding to the passage of the TPLR-CLC in Canada. It is likely that tobacco companies have made changes to their packages to attract customers, at least in part, as a response to the constraints placed upon them by the TPLR-CLC. For example, an increase in the use of fliptop packaging can be seen as more modern, elegant, or unique, which serves to increase purchasing preference in younger people, especially younger women, who have been shown to prefer smaller packaging styles ${ }^{9,10}$. This preference may also explain why we observe that the average number of cigarettes per package has decreased over time. The increased usage of black and yellow as focal colors as well as increased color saturation is likely an effort to attract attention, as research shows that black and yellow are effectively used on tobacco packaging for this purpose ${ }^{12,13}$. Moreover, these attributes may serve 
to draw attention away from the unsightly health labels and to differentiate the brand. The increased usage of brand variant labels and female figures on packaging may be an effect of strategies pursued by tobacco companies to influence positive consumer perceptions of their products in light of increasingly restrictive packaging regulations. The decreased usage of males in iconography suggests that manufacturers may be targeting women. However, it is important to note that these findings are correlational in nature. It is possible that tobacco companies naturally innovate their package design to better market their products, and we cannot be absolutely certain that all of these observed changes were caused by the implementation of the TPLR-CLC.

\section{CONCLUSIONS}

The results of the present study provide evidence of systematic changes to tobacco packaging corresponding to the passage of the TPLR-CLC in Canada. The observed changes include: an increase in the use of flip-top style packaging; white, black and yellow as focal colors; blue, red and yellow as secondary colors; overall color saturation and colorthemed variant labels; a decrease in the number of cigarettes per package; and use of iconography containing male figures. We hope that these findings supplement other research demonstrating that tobacco manufactures alter elements of package design as a function of regulatory changes.

\section{REFERENCES}

1. World Health Organization. WHO report on the global tobacco epidemic, 2017: monitoring tobacco use and prevention policies. https://www.who.int/tobacco/ global_report/2017/en/. Published July 19, 2017. Accessed May 1, 2019.

2. Wakefield M, Morley C, Horan, JK, Cummings KM. The cigarette pack as image: new evidence from tobacco industry documents. Tob Control. 2002;11(Suppl I):i73-i80. doi:10.1136/tc.11.suppl_1.i73

3. Hammond D, Fong GT, McDonald PW, Cameron R, Brown KS. Impact of the graphic Canadian warning labels on adult smoking behaviour. Tob Control. 2003;12(4):391395. doi:10.1136/tc.12.4.391

4. Justice Laws Website. Tobacco Products Labelling Regulations (Cigarettes and Little Cigars) (SOR/2011177). https://laws-lois.justice.gc.ca/eng/regulations/ sor-2011-177/FullText.html. Published September 22, 2011. Accessed March 19, 2019.

5. Canada Gazette. Regulatory impact analysis statement. http://www.gazette.gc.ca/rp-pr/p1/2011/2011-02- 19/html/reg3-eng.html. Published February 19, 2011. Accessed March 28, 2019.

6. Government of Canada. Canadian Tobacco, Alcohol, and Drugs Survey (CTADS): 2015 summary. https://www. canada.ca/en/health-canada/services/canadian-tobaccoalcohol-drugs-survey/2015-summary.html. Accessed November 14, 2018.

7. Government of Canada. Canadian Tobacco, Alcohol, and Drugs Survey (CTADS): summary of results for 2017. https://www.canada.ca/en/health-canada/services/ canadian-tobacco-alcohol-drugs-survey/2017-summary. html. Accessed November 14, 2018.

8. Janz T. Current smoking trends. Statistics Canada Website. http://www.statcan.gc.ca/pub/82-624-x/2012001/ article/11676-eng.htm. Accessed November 14, 2018.

9. Hammond D. Health warning messages on tobacco products: a review. Tob Control. 2011;20(5):327-337. doi:10.1136/tc.2010.037630

10. Kotnowski K, Hammond D. The impact of cigarette pack shape, size and opening: evidence from tobacco company documents. Addiction. 2013;108(9):1658-1668. doi:10.1111/add.12183

11. Moodie C, Ford A. Young adult smokers' perceptions of cigarette pack innovation, pack colour and plain packaging. Australia's Market J. 2011;19(3):174-180. doi:10.1016/j.ausmj.2011.05.003

12. Hammond D. Tobacco packaging and labeling policies under the US Tobacco Control Act: research needs and priorities. Nicotine Tob Res. 2011;14(1):62-74. doi:10.1093/ntr/ntr182

13. Lempert L, Glantz S. Implications of tobacco industry research on packaging colors for designing health warning labels. Nicotine Tob Res. 2016;18(9):1910-1914. doi:10.1093/ntr/ntw127

\section{ACKNOWLEDGEMENTS}

The authors thank Health Canada for supplying the tobacco packages that were used in this research.

\section{CONFLICTS OF INTEREST}

The authors have each completed and submitted an ICMJE form for disclosure of potential conflicts of interest. The authors declare that they have no competing interests, financial or otherwise, related to the current work. Both authors report personal fees and non-financial support from Health Canada, during the conduct of the study. Health Canada also provided the cigarette packages for the research. We note that they did not influence the conclusions of the research in any way.

\section{FUNDING}

This research was supported by Health Canada.

AUTHORS' CONTRIBUTIONS

WSW conducted most of the data analysis for the study. WSW and KW drafted and revised the manuscript.

PROVENANCE AND PEER REVIEW

Not commissioned; externally peer reviewed. 